\title{
A Rare Case of Isolated Transverse Palatal Fracture- A Case Report
}

\author{
Shiney Dominic ${ }^{1}$, R Dibin ${ }^{2}$, D Annapoorani ${ }^{3}$, P K Alshakhir ${ }^{4}$, \\ Muhammad Ali Thayyullathil ${ }^{5}$ \\ Department OfOral And Maxillofacial Surgery, Government Dental College, Kottayam, Kerala, India-686008, \\ $2,3,4$
}

Abstract: Palatal fractures generally occur in conjunction with maxillary fractures. They rarely occur as an isolated fracture and can be easily overlooked by an inexperienced clinician as a dentoalveolar fracture. So it's imperative to have a thorough clinical and radiographic evaluation to diagnose these types of fractures. This article reports a caseof isolated transverse palatal fracture and its management.

Keywords: lefort 1 fracture, maxillary fractures, palatal fractures

\section{Introduction}

The midfacial skeleton is constructed by primarily of maxilla, palate, nasal bones, and zygomas. The greatest portion of the middle third of the face is built by maxilla, which is attached to cranium and supported by strong buttresses. It articulates with most facial bones, including the frontal, ethmoid, nasal, zygomatic, lacrimal, and palatine bones ${ }^{[1]}$. Of these many articulations, some are thin, delicate bone. However, the palatine process of the maxilla, or hard palate, is an exception owing to its thick and sturdy osseous stock. Maxillary fractures most often occur in conjunction with other facial fractures and are most often associated with injuries such as lacerations, other facial fractures, orthopaedic injury and neurologic injury ${ }^{[2]}$. Palatal fractures are relatively rare but generally occur along with maxillary fractures. The average incidence of palatal fractures combined with maxillary fractures ranges from $8 \%$ to $20 \%{ }^{[3-6]}$, although a much higher incidence has recently been reported $(46.4 \%)^{[7]}$

Although various techniques in managementhave been described in the literature, ranging from invasive open reduction and internal fixation (ORIF),Kirschner wire fixation,tonon-invasive techniques such as stabilization of the maxillary arch using an arch bar, transpalatal wiring,${ }^{[8]}$ intraosseous wiring, acrylic splints,andintermolarwiring,all have inherent difficulties and drawbacks.

\section{Case Report}

A 59-year-old male was involved in a motor vehicle accident and reported that he hit his lower jaw in the ground. He was brought to casuality complaining that he has severe pain in the throat region and feeling of nausea. The patient was fully awake conscious and he clinically presented with lacerated wound in the forehead, chin region and intra orally in the labial mucosa of lower lip. The patient was partially edentulous with chronic generalised periodontitis and multiple root stumps on the maxillary arch and mandibular arch. The lacerated wound was sutured using vicryl and nylon sutures. On examination bilateral dentoalveolar fracture including 16, 17, 18 and 27, 28 was found (fig 1 and 2). The bleeding was controlled and CT head and facial bone advised.

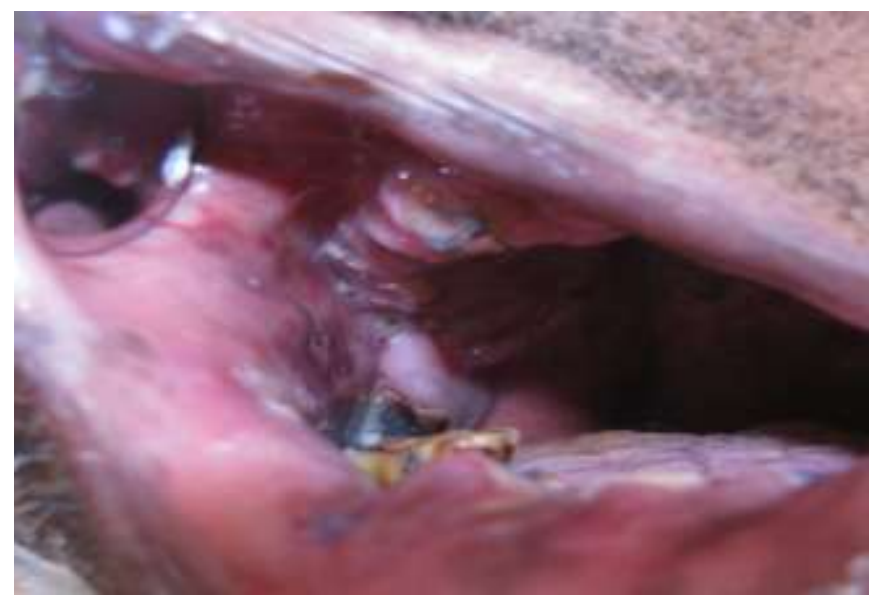

Fig. 1- Right dentoalveolar fracture 


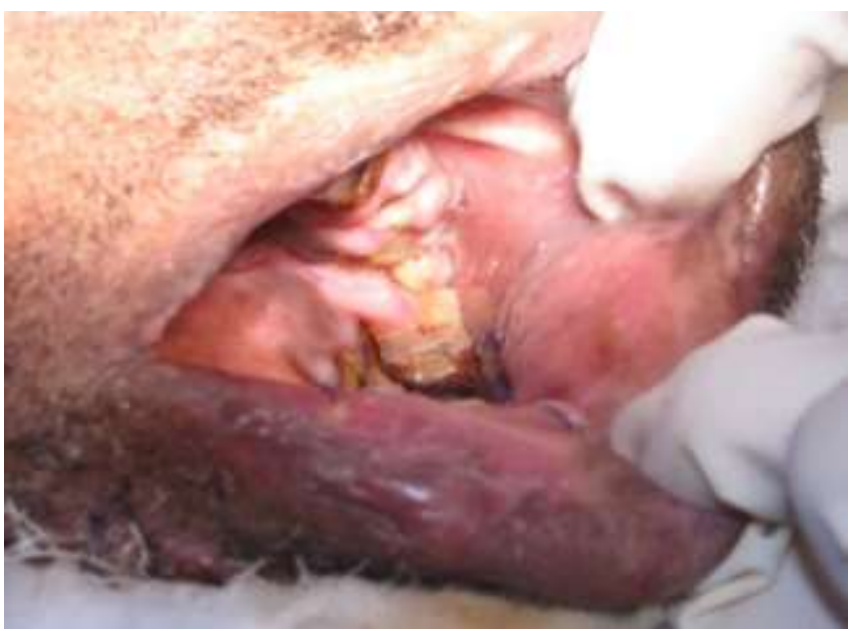

Fig. 2-Left dentoalveolar fracture

CT scan revealed hemosinus, bilateral maxillary sinus wall fracture, lateral pterygoid plate fracture, and nasal septalfracture( fig 3). CT shows a transversely running fracture line in the posterior third of hard palate(fig 4). No fracture was noticed in the mandibular arch.The patient's medical history revealed uncontrolled diabetes and a recent attack of myocardial infarction 2 months back. The patient was managed conservatively with crepe bandage, and intra venous antibiotics and analgesics. The patient was reviewed in one week's interval time for the first one month followed by 2 weeks in the subsequent months. There was uneventful healing of the posterior palatal fracture and the dentoalveolar segments. After a period of one year total extraction was carried out for the patient and prosthethic rehabilitation was done.

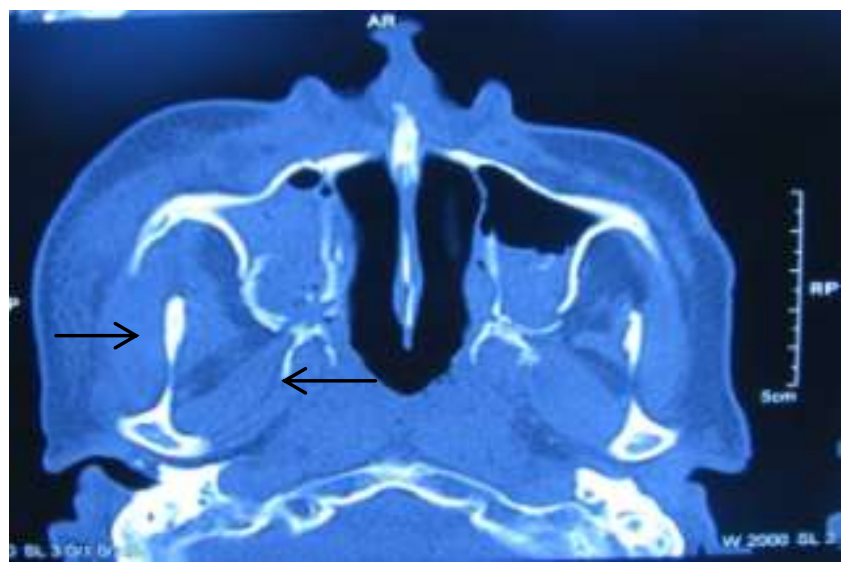

Fig. 3- CT showing bilateral maxillary sinus wall fracture, lateral pterygoid plate fracture, and nasal septal fracture

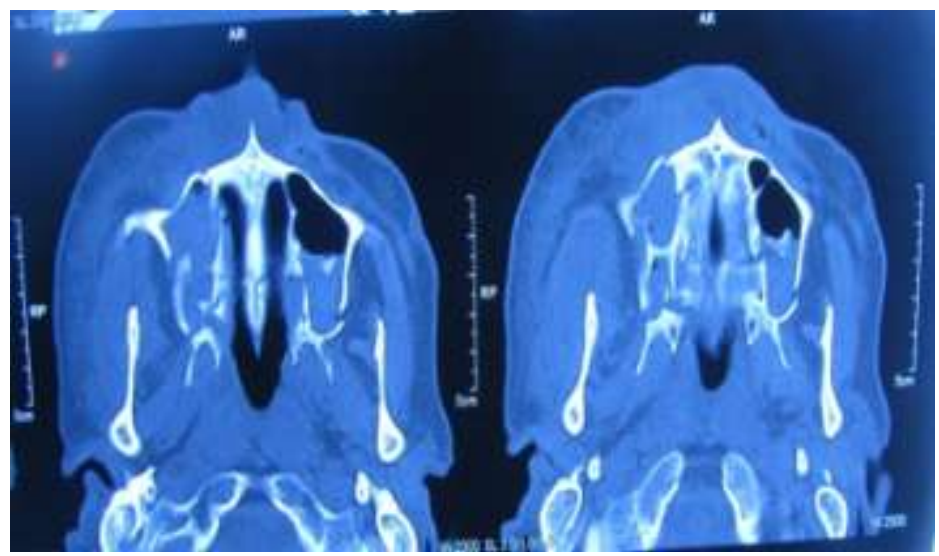

Fig. 4- Transversely running fracture line in the posterior third of hard palate 


\title{
III. Discussion
}

Palatal fractures occur in approximately $8 \%$ of LeFort fractures. They rarely occur as an isolated fracture. A palatal fracture should be expected when there is disruption of the palatal and gingival mucosa. Other clinical signs may include a change in occlusion, mobility of alveolar segments, or a palpable bony step in the palatal vault. A CT scan with axial and coronal slices should be acquired to confirm the diagnosis.

\author{
(A)TypeIa, Anterior alveolus; \\ (B)TypeIb, Posterolateral alveolus; \\ (C)Type II, Sagittal; \\ (D)Type III, Parasagittal; \\ (E)Type IV, Para-alveolar; \\ (F)Type V, Complex/comminuted; and \\ (G)Type VI, Transverse
}

Hendrickson $M$ et al classified palatal fractures ${ }^{[4]}$

Treatment of palatal fractures may involve a combination of arch bars, rigid internal fixation and palatal or occlusal splints. The goal of treatment is to reproduce the preinjury occlusion. Those fractures involving theanterior alveolus (type Ia) and posterolateral alveolus (type $\mathrm{Ib}$ ) are treated with a segmental arch bar spanning the teeth adjacent to the fracture lines. In addition, when possible, miniplates can be placed in the region of the nasomaxillary buttress (Ia) and zygomaticomaxillary buttress (Ib) to further stabilize these fractures.

A period of 2 to 4 weeks of MMF is recommended. Types II, II, IV, and VI fractures are treated with a combination of arch bars and rigid internal fixation. Comminuted (type V) fractures are treated with a palatal acrylic splint ${ }^{[9]}$. Postoperatively, patients should be kept on a soft diet for 4 to 6 weeks. When arch bars are in place, guiding elastics can be used after MMF is released ${ }^{[9]}$.

As the application of arch bar was not feasible in the present case. The patient was put on crepe bandage and soft diet for a period of four weeks. There was uneventful healing of the posterior palatal fracture and the dento-alveolar segments. After a period of one year total extraction was carried out and prosthethic rehabilitation was done.

In summary, transverse palatal fracture alone is rare and can be overlooked by an inexperienced clinician. It can be diagnosed with a thorough clinical and radiographic examination. Based on the literature, reduction should be attempted as soon as the diagnosis is made with arch bar followed byminiplate fixation if possible. This should be followed by a short period of intermaxillary fixation (about 2 weeks). Associated mandibular and midfacial fractures should determine the approach and sequencing of fracture reduction and period of intermaxillary fixation to achieve preinjury occlusion.

\section{Conclusion}

Palatal fracture is often overlooked and is the cause of post-operative malocclusion in trauma patients. Often they occur in association with lefort fractures. Isolated transverse fractures were rare so a thorough clinical and radiographic evaluation is essential to diagnose these types of fractures. Closed reduction was done as a treatment modality in majority of patients with palatal bone fractures.

\section{References}

[1]. Manson PN, Clark N, Robertson B, et al: Subunit principles in midface fractures: The importance of sagittal buttresses, softtissue reductions, and sequencing treatment of segmental fractures. PlastReconstrSurg 103:1287, 1999

[2]. Leech TR, Martin BC, Trabue JC. An analysis of the etiology, treatment and complications of fractures of the malar compound and zygoma. J Surg 1956;92;920-924

[3]. Denny AD, Celik N: A management strategy for palatal fractures:A 12-year review. J CraniofacSurg 10:49, 1999

[4]. Hendrickson M, Clark N, Manson PN, et al: Palatal fractures: Classification, patterns, and treatment with rigid internal fixation. PlastReconstrSurg 101:319, 1998

[5]. Park S, Ock JJ: A new classification of palatal fracture and an algorithm to establish a treatment plan. PlastReconstrSurg 107:1669, 2001

[6]. Manson PN, Shack RB, Leonard LG, et al: Sagittal fractures of the maxilla and palate. PlastReconstrSurg 72:484, 1983

[7]. Chen CH, Wang TY, Tsay PK, et al: A 162-case review of palatal fracture: Management strategy from a 10-year experience. PlastReconstrSurg 121:2065, 2008

[8]. Davis DG, Constant E: Transverse palatal wire for the treatment of vertical maxillary fractures. PlastReconstrSurg 48:191, 1971

[9]. Atlas Oral Maxillofacial SurgClin N Am 21 (2013) 69e95 1061-3315/13/\$ 